\title{
Effect of hyperventilation and mental stress on coronary blood flow in syndrome $\mathrm{X}$
}

\author{
A Chauhan, P A Mullins, G Taylor, M C Petch, P M Schofield
}

\begin{abstract}
Objectives-To assess the effect of hyperventilation and mental stress on coronary blood flow and symptom production in patients with syndrome $X$.

Design-A prospective study. Hyperventilation and mental stress tests were performed on the ward and were repeated in the cardiac catheter laboratory where coronary blood flow velocity was also measured with an intracoronary Doppler catheter in the left anterior descending coronary artery. Oesophageal manometry studies were also performed.
\end{abstract}

Patients-29 patients with syndrome $X$ (typical anginal chest pain, a positive exercise test, and normal coronary angiogram).

Setting-A regional cardiothoracic centre. Results-Hyperventilation produced typical chest pain in 16 patients on the ward. 13 patients experienced their typical chest pain with mental stress test 5 . Ten patients experienced chest pain with both hyperventilation and mental stress tests. This pattern was reproduced exactly when the tests were repeated in the cardiac catheter laboratory. Hyperventilation produced a significant increase in the rate-pressure product during ward and laboratory testing. There was, however, no significant change in the ratepressure product on mental stress tests. The mean (SEM) coronary flow velocity decreased significantly on hyperventilation in the catheter laboratory from 10.0 $(0.92) \mathrm{cm} / \mathrm{s}$ to $5.9(0.72) \mathrm{cm} / \mathrm{s}(p<0.001)$. There was also a significant reduction in the mean (SEM) coronary blood flow velocity on mental stress tests from 9.8 $(0.86) \mathrm{cm} / \mathrm{s}$ to $7.4(0.6) \mathrm{cm} / \mathrm{s}(p<0.001)$. This reduction in flow velocity occurred in the absence of any changes in diameter of the left anterior descending artery. Further analysis showed that the coronary flow velocity was reduced significantly in only that group of patients in which hyperventilation and mental stress provoked chest pain. There was a significant increase in the arterial concentrations of noradrenaline on both hyperventilation and mental stress testing. Oesophageal manometry showed abnormalities in $17 \%$ of patients.

Conclusions-Both hyperventilation and mental stress can produce chest pain in patients with syndrome $X$ and this is associated with a reduction in coronaryo blood flow velocity. The results of this $\bar{D}$ study suggest that this reduction in coro- $-\overline{\bar{c}}$. nary flow occurs as a result of increased $\frac{\Phi}{\complement}$ microvascular resistance.

(Br Heart $\mathcal{f} 1993 ; 69: 516-524)$

In more than $10 \%$ of patients undergoing coronary angiography for the investigation of chest pain the coronary angiogram is normal. ${ }^{12}$ These patients have chest pain and $\frac{\vec{\sigma}}{2}$ abnormal changes on the exercise electrocar-o diogram and are said to have "angina pectoris? with normal coronary angiograms" or "syn- $c$ drome X". The term syndrome $X$ was first used by Kemp in his editorial comment on a paper by Arbogast and Bourassa in which heco noted the heterogeneity of patients included in studies of patients with chest pain and nor-O mal coronary angiograms and the uncertainty of the aetiology of chest pain in these patients. ${ }^{3}$ Subsequently, the term became a\% label for patients with typical angina and normal coronary angiograms although investiga $-\overrightarrow{\overrightarrow{0}}$ tors have often used different definitive ${ }_{\supset}^{\exists}$ criteria.

The mechanism of chest pain in patients with syndrome $\mathrm{X}$ is not clear. Many explana- $\mathrm{D}$ tions have been put forward to explain this? syndrome but as more information has become available the syndrome has become more confusing. It has been suggested that some patients with syndrome $\mathrm{X}$ may evolve towards dilated cardiomyopathy suggesting? that in a subset of patients a cardiomyopathico process may play a pathogenic part. ${ }^{4}$. Metabolic abnormalities as an index of myocardial ischaemia have been reported in N patients with syndrome $\mathrm{X} .{ }^{67}$ Misinterpreta- ${ }_{\mathrm{C}}$ tion of the coronary angiograms, ${ }^{8}$ oxyhaemoglobin dissociation defects, ${ }^{9}$ psychosomati\&o

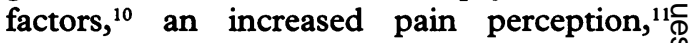
oestrogen deficiency, ${ }^{12}$ and abnormalities of ${ }^{\circ}$ the potassium pump ${ }^{13}$ have all been suggested as possible explanations for syndrome $\mathrm{X}$. $\mathrm{An} \frac{\mathrm{C}}{\mathrm{D}}$ abnormal coronary flow reserve has also been $\frac{\rho}{\Phi}$ shown in syndrome $\mathrm{X}$ by different methods $\cong$ and by several investigators. This has been reviewed by Cannon et al. ${ }^{14}$ The proposed mechanisms for abnormalities of coronary flow reserve have included structural abnor malities of the microcirculation, ${ }^{15}$ increased? sympathetic drive affecting both epicardial and small coronary vessels, ${ }^{16}$ endothelial dysfunction, ${ }^{17}$ and hyperinsulinaemia. ${ }^{18}$ Recently it has been suggested that the fundamental 
abnormality in syndrome $\mathrm{X}$ might be an increased tone of the prearteriolar coronary vessels interposed between the conductance arteries and the arterioles and this may account for the reduced coronary vasodilatory capacity of the microcirculation. ${ }^{1920}$ It has now become clear that syndrome $\mathrm{X}$ is a heterogeneous entity and therefore it is unlikely that a unique pathophysiological mechanism is responsible for its clinical presentation and prognosis. Kemp had postulated in his original editorial that mechanisms may be different in different patients encompassing several pathophysiological disease entities and this view has now become widely accepted. ${ }^{3}$

Hyperventilation, anxiety, and stress can also produce chest pain in patients with syndrome $X .^{21}$ The mechanism by which these factors can produce chest pain and electrocardiographic changes is not clear. A diffuse abnormal epicardial coronary artery constrictor response to hyperventilation and other constrictor stimuli has been suggested. ${ }^{22} \mathrm{We}$ investigated the hypothesis that hyperventilation and mental stress can produce chest pain in patients with syndrome $\mathrm{X}$ by affecting coronary blood flow.

\section{Patients and methods \\ PATIENTS}

We studied the effect of hyperventilation and mental stress in a group of 29 patients with syndrome $\mathrm{X}$. All patients gave a history of chest pain typical of angina pectoris. There was no evidence of cardiovascular disease on physical examination. The exercise electrocardiogram was positive in every patient. The Bruce protocol was used for the exercise test and the test was said to be positive if there was at least $1 \mathrm{~mm}$ of horizontal or downward sloping ST segment depression at $80 \mathrm{~ms}$ after the J point. The left ventricle and the coronary arteries were completely normal on angiography and this was confirmed by two independent observers. Patients with hypertension, diabetes mellitus, and valvar heart disease or left ventricular hypertrophy were excluded from the study. All patients had continued to have chest pain despite reassurances after their initial cardiac catheterisation and were taking antianginal medications. The study was approved by the Huntingdon District Health Authority Ethics Committee as part of a coronary flow study and all patients gave written informed consent before the study.

\section{ECHOCARDIOGRAPHY}

The left ventricular posterior wall and septal thickness of all patients was assessed by cross sectional and $M$ mode echocardiography. Patients with a diastolic septal or posterior wall thickness of more than $11 \mathrm{~mm}$ were excluded from the study to minimise any effect of left ventricular hypertrophy on coronary blood flow measurements.

BLOOD ANALYSIS

Patients' blood was analysed for full blood count, serum urea and electrolytes, and fasting lipids on the morning of their cardiac catheter study.

\section{STUDY PROTOCOL}

Patients first underwent hyperventilation and mental arithmetic stress tests on the ward and these were repeated in the cardiac catheter laboratory 24 hours later when coronary blood flow velocity, was also measured with an intracoronary Doppler catheter. All patients underwent oesophageal manometry to assess the prevalence of oesophageal dysfunction.

\section{Hyperventilation protocol}

Patients were instructed to breathe rapidly (rate $>30$ breaths /minute) for five minutes. Electrocardiogram and blood pressure was recorded at rest, at one minute intervals during the test, and for 10 minutes after the test. Patients were instructed to report any chest pain and to distinguish if this was their usual chest pain.

\section{Mental arithmetic stress protocol}

Patients were asked to subtract sequentially 13 from 1017. If they could not manage these numbers, they were asked to subtract sequentially seven from 113. The duration of the test was three minutes. Electrocardiogram and blood pressure were recorded at rest, at one minute intervals during the test, and for five minutes after the test. Patients were instructed to report any chest pain and to distinguish if this was their usual chest pain. Patients were prompted periodically to increase speed and accuracy. This task is a standard cognitive mental challenge.

The hyperventilation test was followed by the mental arithmetic stress test in the first 10 patients and the tests were reversed in the remaining 19. At least 10 minutes were allowed to lapse in between the tests. No coffee or smoking was allowed on the day of the test.

Tests on the ward

All cardiac medication was stopped at least 24 hours before the test. The electrocardiogram was recorded with a Hewlett Packard 4745A Pagewriter II recorder.

\section{Tests in the catheter laboratory}

The tests were performed 24 hours after the tests on the ward. All cardiac medications had been stopped for at least 48 hours. Patients were given $10 \mathrm{mg}$ diazepam as premedication before cardiac catheterisation. A standard right femoral approach was used. Sodium heparin (10 000 U) was given intravenously. An $8 \mathrm{~F}$ guide catheter was positioned in the left coronary ostium. Through this a 0.014 inch guidewire was advanced into the distal part of the left anterior descending coronary artery. With a monorail technique, a $3.6 \mathrm{~F} 20 \mathrm{MHz}$ intracoronary Doppler flow catheter (Schneider, UK) was then advanced over the guide wire and positioned in the proximal segment of the left 
anterior descending coronary artery. This was connected to a Millar velocimeter (Model MDV-20, Millar Instruments, Houston, Texas) that was range gated and calibrated (1 $\mathrm{kHz}=3.75 \mathrm{~cm} / \mathrm{s}$ ). The Doppler flow catheter and the range gate of the velocimeter were adjusted to obtain good quality phasic and mean signals of coronary blood flow velocity. These signals were recorded on a Mingograf recorder (Siemens-Elema, Sweden). Coronary blood flow velocity was measured at rest with the patient breathing normally, and immediately before and after the hyperventilation, and mental stress tests.

\section{CORONARY ANGIOGRAPHY}

Coronary angiograms of the left anterior descending artery were taken in preselected views, at rest and after the hyperventilation and mental stress tests immediately measurement after coronary blood flow velocity had been recorded.

\section{QUANTITATIVE MEASUREMENTS}

The diameter of the left anterior descending coronary artery was measured with digital electronic callipers (Sandhill Scientific Inc). This method has been used previously to assess the arterial diameter of coronary vessels. $^{23} 24$ Two selected views were taken and projected by a Tagarno system on to a sheet of paper. The arterial diameter of the left anterior descending coronary artery was measured from tracings of the projected images in diastole, at $3 \mathrm{~cm}$ from the tip of the Doppler catheter. The diameter was calculated as the mean of the measurements of the two views. Two independent observers make the measurements so that inter and intra observer variation could also be calculated.

\section{CATECHOLAMINE MEASUREMENTS}

Blood samples were taken from the aortic root before and after the hyperventilation and mental stress tests. Adrenaline and noradrenaline concentrations were measured by high performance liquid chromatography. ${ }^{25}$

\section{ARTERIAL BLOOD GAS MEASUREMENTS}

Samples were taken from the aortic root for $\mathrm{pH}$ and $\mathrm{PCO}_{2}$ before and after the hyperventilation test.

\section{OESOPHAGEAL FUNCTION TESTS}

All cardiac medications had been discontinued at least 24 hours before the study. Most of the oesophageal function studies were performed in the morning before cardiac catheterisation. After an overnight fast, lignocaine topical anaesthetic was sprayed into the nasopharynx. A multisensor catheter (diameter $3.5 \mathrm{~mm}$ ) was passed into the oesophagus. This was connected to a GR 800 analysing station and signals were displayed on a screen and stored in the computer for analysis. Peristaltic activity was assessed with five wet and dry swallows, each separated by a 30 s interval. Patients were asked to report any chest pain and to distinguish whether the pain was typical or atypical of their usual pain. After the baseline study, edrophonium hydrochloride $(80 \mu \mathrm{g} / \mathrm{kg})$ was given intravenously followed immediately by five wet swallows. Manometric responses were recorded for later analysis.

CRITERIA FOR ABNORMAL OESOPHAGEAL FUNCTION

Studies were performed and interpreted by an independent investigator. The criteria for a $\stackrel{c}{\mathrm{c}}$ diagnosis of oesophageal dysfunction were:

(a) Achalasia: aperistalsis of the oesophageal body with incomplete lower oeso- $\frac{}{c}$ phageal sphincter relaxation and increased $\frac{\bar{\sigma}}{\bar{D}}$.

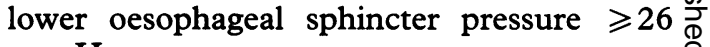
$\mathrm{mm} \mathrm{Hg}$.

(b) Nutcracker oesophagus: mean peristaltic amplitude measured with the catheter $\overrightarrow{0}$

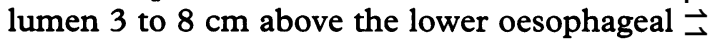
sphincter) $\geqslant 180 \mathrm{~mm} \mathrm{Hg}$ averaged over five $\stackrel{\omega}{\omega}$ wet swallows.

(c) Diffuse oesophageal spasm: repetitive, spontaneous, non-peristaltic contractions in at least $30 \%$ of contractions, with otherwise or normal peristalsis.

(d) Non-specific motility disorder: pro- 은 longed duration of oesophageal contraction $7 \overrightarrow{-}$

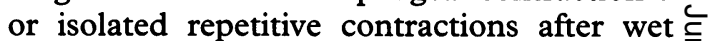
swallows.

STATISTICAL ANALYSIS

The Wilcoxon signed rank test for paired data was used for statistical evaluation. Differences were considered to be significant at the $\mathrm{p}<0.05$ level. All data are presented as mean value (SEM).

\section{Results}

Table 1 shows relevant information about patients. There were 11 men and 18 women. The mean age was 53 (range 23-67) years. The mean exercise test duration was eight $\frac{7}{\circ}$ minutes and 20 seconds (range four minutes 3 . and 40 seconds to 12 minutes). All patients were receiving anti anginal medications. Ten $₹$ patients were taking $\beta$ blockers, 15 calcium 웅 antagonists, and 12 oral nitrates. Twelve patients were also taking oral aspirin.

Table 1 Patient variables (mean (SEM))

\begin{tabular}{|c|c|}
\hline Variable & Value \\
\hline \multirow{2}{*}{\multicolumn{2}{|c|}{ Sex: }} \\
\hline & \\
\hline Men & 11 \\
\hline Women & 18 \\
\hline Weight (kg) & $73(2 \cdot 1)$ \\
\hline Smokers & 10 \\
\hline $\mathrm{Hb}(\mathrm{g} / \mathrm{dl})$ & $13.2(0.4)$ \\
\hline $\mathrm{PCV}(1 / 1)$ & $0.41(0.01)$ \\
\hline Urea $(\mathrm{mmol} / \mathrm{l})$ & $7 \cdot 12(0 \cdot 64)$ \\
\hline Glucose $(\mathrm{mmol} / \mathrm{l})$ & $5.6(0.2)$ \\
\hline Creatinine $(\mu \mathrm{mol} / \mathrm{l})$ & $119(7 \cdot 7)$ \\
\hline Cholesterol $(\mathrm{mmol} / 1)$ & $6 \cdot 4(0 \cdot 2)$ \\
\hline $\mathrm{HDL}(\mathrm{mmol} / \mathrm{l})$ & $0.98(0.06)$ \\
\hline $\mathrm{LDL}(\mathrm{mmol} / \mathrm{l})$ & $4.31(0 \cdot 2)$ \\
\hline Triglycerides $(\mathrm{mmol} / \mathrm{l})$ & $1.97(0.14)$ \\
\hline LVEDP $(\mathrm{mm} \mathrm{Hg})$ & $8(0.8)$ \\
\hline \multicolumn{2}{|l|}{ Antianginal drugs: } \\
\hline Nitrates & 12 \\
\hline Calcium antagonists & 15 \\
\hline$\beta$ blockers & 10 \\
\hline
\end{tabular}

HG, haemoglobin; PCV, packed cell volume; HDL, high density lipoprotein; LDL, low density lipoprotein; LVEDP, left ventricular end diastolic pressure. 
CHEST PAIN

Sixteen patients experienced their typical angina like chest pain during the hyperventilation test on the ward and 13 patients during mental stress. Ten patients experienced chest pain with both hyperventilation and mental stress. There were, however, no significant ST segment changes on the electrocardiogram. When the tests were repeated in the cardiac catheter laboratory 24 hours later this pattern of chest pain was reproduced exactly in the same patients. Once again the episodes of chest pain were not associated with any significant ST segment changes on the electrocardiogram.

\section{HAEMODYNAMICS}

Most patients experienced light headedness or tingling by two minutes of the hyperventilation test. In the hyperventilation test on the ward there was a significant increase in the heart rate $(p<0.001$ table 2$)$. There was no significant change in systolic blood pressure. The significant increase in the rate-pressure product was a reflection of the increase in heart rate $(p<0.001)$. Hyperventilation test in the cardiac catheter laboratory again produced a significant increase in the heart rate ( $p<0.001$ table 3 ) without a significant change in the systolic blood pressure. The significant increase in the rate-pressure product again reflected the increase in heart rate $(\mathrm{p}<0.001)$.

With a mental stress test on the ward the heart rate did not increase significantly and there was no significant change in the systolic blood pressure. As a result there was no significant change in the rate-pressure product (table 2). This pattern was repeated in the stress tests performed in the catheter laboratory (table 3).

Table 2 Results of ward hyperventilation and mental stress tests (mean (SEM))

\begin{tabular}{lrrl}
\hline & \multicolumn{1}{l}{ Before } & \multicolumn{1}{l}{ After } & \multicolumn{1}{l}{$p$ Value } \\
\hline Ward hyperventilation: & & & \\
Heart rate (beats/min) & $68(3 \cdot 9)$ & $80(4 \cdot 2)$ & $<0 \cdot 001$ \\
Systolic pressure (mm Hg) & $136(4 \cdot 5)$ & $138(5 \cdot 0)$ & NS \\
Rate-pressure product & $9248(560)$ & $11040(650)$ & $<0 \cdot 001$ \\
Ward stress: & $70(3 \cdot 2)$ & $76(3 \cdot 6)$ & NS \\
Heart rate (beats/min) & $132(4 \cdot 4)$ & $134(4 \cdot 6)$ & NS \\
Systolic pressure (mm Hg) & $9240(556)$ & $10184(640)$ & NS \\
Rate-pressure product & & &
\end{tabular}

Table 3 Catheter laboratory results (mean (SEM))

\begin{tabular}{lccl}
\hline & Before & After & $p$ Value \\
\hline Hyperventilation test: & & & \\
Heart rate (beats/min) & $72(3 \cdot 2)$ & $84(4 \cdot 1)$ & $<0 \cdot 001$ \\
Systolic pressure (mm Hg) & $130(4 \cdot 2)$ & $128(4 \cdot 6)$ & NS \\
Mean arterial pressure (mm Hg) & $101(2 \cdot 2)$ & $100(2 \cdot 1)$ & NS \\
Rate-pressure product & $8681(657)$ & $9828(774)$ & $<0 \cdot 001$ \\
Coronary flow velocity (cm/s) & $10 \cdot 0(0 \cdot 92)$ & $5 \cdot 9(0 \cdot 72)$ & $<0 \cdot 001$ \\
Adrenaline (ng/l) & $60(7)$ & $74(9)$ & NS \\
Noradrenaline (ng/l) & $277(22)$ & $379(38)$ & 0.005 \\
Stress test: & $73(3 \cdot 4)$ & $76(3.5)$ & 0.06 \\
Heart rate (beats/min) & $137(4 \cdot 5)$ & $137(4 \cdot 9)$ & NS \\
Systolic pressure (mm Hg) & $104(2 \cdot 9)$ & $102(2 \cdot 9)$ & NS \\
Mean arterial pressure (mm Hg) & $8985(789)$ & $9331(839)$ & $<0 \cdot 001$ \\
Rate-pressure product & $9 \cdot 8(0 \cdot 86)$ & $7 \cdot 4(0 \cdot 6)$ & $<0 \cdot 001$ \\
Coronary flow velocity (cm/s) & $60(7)$ & $65(6)$ & NS \\
Adrenaline (ng/l) & $297(25)$ & $359(35)$ & 0.002 \\
Noradrenaline (ng/l) & & & \\
\hline
\end{tabular}

CORONARY BLOOD FLOW VELOCITY

The mean, coronary blood flow velocity decreased significantly on hyperventilation in the catheter laboratory from $10.0(0.92) \mathrm{cm} / \mathrm{s}$ to $5.9(0.72) \mathrm{cm} / \mathrm{s}(\mathrm{p}<0.001$, table 3$)$. There was also a significant reduction in the mean coronary blood flow velocity on mental stress tests from $9.8(0 \cdot 86) \mathrm{cm} / \mathrm{s}$ to $7 \cdot 4(0.6) \mathrm{cm} / \mathrm{s}$ $(\mathrm{p}<0.001$, table 3$)$. In all cases the coronary blood flow velocity returned to normal within five minutes of stopping the tests although some of the patients continued to experience chest pain for some time after that.

We further analysed the results of the hyperventilation and mental stress tests by comparing the results of the changes in coronary flow velocity, in patients in whom the tests produced chest pain with the results from those patients who did not experience chest pain. There were no significant differences between the groups with respect to their clinical characteristics and drug use. In patients who experienced their typical chest pain on hyperventilation there was a significant reduction in velocity of coronary blood flow $(11.1(1.2) v 5.0(0.9) \mathrm{cm} / \mathrm{s} \mathrm{p}<0.001$, table 4). Hyperventilation, however, did not significantly affect the coronary blood flow velocity in patients who did not experience any chest pain $(8.7(1.4)$ v $7.0(1.1) \mathrm{cm} / \mathrm{s}$, NS, table 4). In patients in whom mental stress produced their typical chest pain, there was also a significant reduction in coronary blood flow velocity $(10.7(1.4) v 6.0(0.6)$ $\mathrm{cm}, \mathrm{p}=0.002$, table 5) whereas there was no effect on the coronary blood flow velocity in patients not experiencing any chest pain $(9 \cdot 1$ $(1.0) v 8.6(0.9) \mathrm{cm} / \mathrm{s}, \mathrm{NS}$, table 5).

\section{ARTERIAL BLOOD GAS ANALYSIS}

The $\mathrm{pH}$ on hyperventilation increased from $7.42(0.005)$ to $7.58(0.006)$. The $\mathrm{PCO}_{2}$ decreased from $35(0.9)$ to $21(0.6) \mathrm{mm} \mathrm{Hg}$, indicating effective hyperventilation.

\section{CATECHOLAMINE MEASUREMENTS}

There was a significant increase in the arterial concentrations of noradrenaline after hyperventilation and mental stress tests (table 3 ). There was no significant change in the arterial adrenaline concentrations after these tests when the results were analysed for the entire group. Analysis of the results by comparing patients who experienced chest pain with those who did not showed that the noradrenaline concentrations were increased by hyperventilation and mental stress tests in both subgroups (tables 4 and 5). There was a significant increase in adrenaline concentrations only in that subgroup of patients who experienced chest pain with hyperventilation (table 4).

\section{DIAMETER OF LEFT ANTERIOR DESCENDING ARTERY}

Quantitative measurements did not show a significant difference in the diameter of the left anterior descending artery before and after the tests (table 6). There was minimal interobserver $(r=0.90)$ and intraobserver variation $(r=0.91)$. 
Table 4 Measurements in patients with and without chest pain on hyperventilation (mean (SEM))

\begin{tabular}{lccl}
\hline & Before & After & p Value \\
\hline Pain on hyperventilation: & & & \\
Heart rate (beats/min) & $77(4 \cdot 5)$ & $90(6 \cdot 3)$ & $0 \cdot 001$ \\
Systolic pressure (mm Hg) & $129(6 \cdot 7)$ & $127(7 \cdot 2)$ & $\mathrm{NS}$ \\
Mean arterial pressure (mm Hg) & $96(4 \cdot 3)$ & $93(6 \cdot 2)$ & $\mathrm{NS}$ \\
Rate-pressure product & $9900(753)$ & $11253(973)$ & $0 \cdot 003$ \\
Coronary flow velocity (cm/s) & $11 \cdot 1(1 \cdot 2)$ & $5 \cdot 0(0 \cdot 9)$ & $<0 \cdot 001$ \\
Adrenaline (ng/1) & $61(9)$ & $83(12)$ & $0 \cdot 05$ \\
Noradrenaline (ng/) & $302(32)$ & $392(30)$ & $0 \cdot 04$ \\
No pain on hyperventilation: & $66(4 \cdot 2)$ & $76(4 \cdot 2)$ & $0 \cdot 002$ \\
Heart rate (beats/min) & $130(4 \cdot 6)$ & $129(5 \cdot 2)$ & $\mathrm{NS}$ \\
Systolic pressure (mm Hg) & $101(2 \cdot 8)$ & $98(3)$ & $\mathrm{NS}$ \\
Mean arterial pressure (mm Hg) & $7942(901)$ & $8938(980)$ & $0 \cdot 004$ \\
Rate-pressure product & $8 \cdot 7(1 \cdot 4)$ & $7 \cdot 0(1 \cdot 1)$ & $0 \cdot 06$ \\
Coronary flow velocity (cm/s) & $59(12)$ & $64(15)$ & $\mathrm{NS}$ \\
Adrenaline (ng/l) & $268(21)$ & $356(40)$ & $0 \cdot 01$ \\
Noradrenaline (ng/l) & & & \\
\hline
\end{tabular}

Table 5 Measurements in patients with and without chest pain on mental stress tests mean (SEM)

\begin{tabular}{lccl}
\hline & Before & After & $p$ Value \\
\hline Pain on stress: & & & \\
Heart rate (beats/min) & $77(4 \cdot 3)$ & $82(6 \cdot 3)$ & $0 \cdot 07$ \\
Systolic pressure (mm Hg) & $136(8 \cdot 6)$ & $39(9 \cdot 5)$ & $\mathrm{NS}$ \\
Mean arterial pressure (mm Hg) & $106(4 \cdot 8)$ & $105(5 \cdot 2)$ & $\mathrm{NS}$ \\
Rate-pressure product & $9704(1206)$ & $10589(1372)$ & $\mathrm{NS}$ \\
Coronary flow velocity (cm/s) & $10 \cdot 7(1 \cdot 4)$ & $6 \cdot 0(0 \cdot 6)$ & $0 \cdot 002$ \\
Adrenaline (ng/l) & $57(7)$ & $72(9)$ & $\mathrm{NS}$ \\
Noradrenaline (ng/l) & $302(39)$ & $415(61)$ & 0.04 \\
No pain on stress: & $71(5 \cdot 3)$ & $72(4 \cdot 9)$ & $\mathrm{NS}$ \\
Heart rate (beats/min) & $138(4 \cdot 5)$ & $135(4 \cdot 6$ & $\mathrm{NS}$ \\
Systolic pressure (mm Hg) & $102(3 \cdot 8)$ & $100(3 \cdot 4)$ & $\mathrm{NS}$ \\
Mean arterial pressure (mm Hg) & $84001051)$ & $8309(1000)$ & $\mathrm{NS}$ \\
Rate-pressure product & $9 \cdot 1(1 \cdot 0)$ & $8 \cdot 6(0 \cdot 9)$ & $\mathrm{NS}$ \\
Coronary flow velocity (cm/s) & $63(13)$ & $60(6)$ & $\mathrm{NS}$ \\
Adrenaline (ng/l) & $270(30)$ & $393(32)$ & $0 \cdot 01$ \\
Noradrenaline (ng/l) & & & \\
\hline
\end{tabular}

Table 6 Measurements of left anterior descending coronary artery diameter mean (SEM))

\begin{tabular}{llll}
\hline & Before & After & $p$ Value \\
\hline Stress & $3.98(0.07)$ & $3.97(0.06)$ & NS \\
Hyperventilation & $4.0(0.04)$ & $4 \cdot 1(0.04)$ & NS \\
\hline
\end{tabular}

OESOPHAGEAL FUNCTION STUDIES

None of the patients experienced chest pain with wet or dry swallows and with the criteria defined already, no patient had any evidence of oesophageal dysfunction. Four patients, however, developed chest pain with evidence of non-specific motility disorder and one had chest pain with associated diffuse oesophageal spasm after an ergonovine provocation test. Thus $17 \%$ of the patients showed evidence of oesophageal dysfunction. The results were analysed further by assessing the prevalence of oesophageal abnormality in patients who experienced chest pain on hyperventilation and mental stress compared with those who did not. All five patients with evidence of oesophageal dysfunction did not experience chest pain on hyperventilation and mental stress tests performed on the ward and in the catheter laboratory.

\section{Discussion}

Our study is the first to report the effects of both hyperventilation and mental stress on coronary blood flow velocity with an intracoronary Doppler catheter in patients with syndrome $\mathrm{X}$. The use of intracoronary Doppler catheters for the measurement of coronary flow velocity has been described in detail elsewhere. ${ }^{26}$ Our study has shown that both hyperventilation and mental stress can produce chest pain in patients with syndrome $\mathrm{X}$ and this is associated with a significant reduction in coronary blood flow velocity in the absence of any significant changes in the diameter of the left anterior descending coronary artery.

Hyperventilation is defined by a minute volume higher than that required for the rate of $\mathrm{CO}_{2}$ production. Neil and Hattenhauer have shown that hyperventilation affects myocardial oxygen supply in humans by a $\frac{\bar{c}}{\bar{N}}$ combination of an increase in the oxygen affinity of the blood due to the Bohr effect, and coronary vasoconstriction that decreases is coronary blood flow. ${ }^{27}$ Ardissino et al have $\overrightarrow{0}$ further suggested that early hyperventilation induced ST segment depression is due to increased oxygen demand as indicated by increase in rate-pressure product, and delayed hyperventilation induced ST segment depression is probably related to a primary or reduction in coronary blood flow. ${ }^{28}$ Syndrome $\vec{\sigma}$

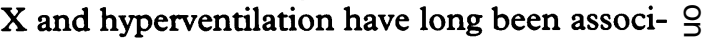
ated. Lewis et al recently reported that patients with syndrome $\mathrm{X}$ do not show inap- $\check{\subseteq}$ propriate hyperventilation in response to $\overparen{D}$ exercise. $^{29}$ Many patients, however, with $\vec{\bullet}$ recurrent hyperventilation do not hyperventi- $ळ$ late during an exercise test but readily overbreathe in response to an emotional challenge. ${ }^{30}$ Our study has shown that forced hyperventilation can produce chest pain associated with a reduction in coronary blood flow velocity in some patients with syndrome $\mathrm{X}$.

In our study effective hyperventilation was confirmed in the catheter laboratory by the increase in arterial $\mathrm{pH}$ and the reduction in $\mathrm{PCO}_{2}$. The number of patients experiencing chest pain on hyperventilation in our study is also consistent with previous studies that have shown that provocation of hyperventilation reproduces pain in about $50 \%$ of patients with chest pain and normal coronary arter- 0 ies. ${ }^{31} 32$ Hyperventilation increased the ratepressure product significantly during tests on the ward and in the catheter laboratory. The $\bar{N}$ coronary blood flow velocity was also reduced $\%$ significantly in the group of patients in whom $N$ hyperventilation provoked chest pain. This $\mathcal{\omega}^{-}$ would seem to support the previously suggested mechanisms that hyperventilation 0 increases myocardial oxygen requirement and $\varnothing_{\overparen{D}}$ also reduces coronary blood flow. It is inter- esting to note that in the group of patients who did not experience chest pain with $\frac{0}{1}$ hyperventilation there was a significant $\stackrel{\odot}{\stackrel{\rho}{\circ}}$ increase in the rate-pressure product without $\varrho$ a significant change in the coronary flow? velocity. This suggests that chest pain may be $\delta$ produced as a result of a dynamic balance between the two mechanisms.

It has been shown that an increase in heart rate increases resting coronary blood flow velocity. ${ }^{33}$ In our study hyperventilation increased the heart rate significantly in both ward and catheter laboratory tests. This 
makes the reduction in coronary blood flow velocity even more important.

Mental stress is known to precipitate angina and myocardial ischaemia in patients with coronary artery disease..$^{34-36}$ Mental stress can affect cardiovascular variables in much the same way as physical exercise, with a common factor being an increase in myocardial oxygen consumption..$^{36-38}$ Thus mental stress may induce myocardial ischaemia in some patients with reduced coronary reserve. ${ }^{39}$ Furthermore, adrenergic activation may increase coronary resistance, attenuating vasodilation caused by the increased oxygen demand and further limiting coronary reserve. ${ }^{40}$ Studies with positron emission tomography in patients with coronary artery disease have shown that mental stress testing can produce an absolute decrease in regional coronary blood flow. ${ }^{34}$ Yeung et al have shown that in patients with coronary atherosclerosis shown by angiography, mental stress can cause myocardial ischaemia not by increasing myocardial oxygen demand but by reducing myocardial flow. ${ }^{41}$ Although the reduction in blood flow was associated with abnormal constriction of atherosclerotic segments of epicardial coronary arteries, the authors concluded that the changes found in the diameter of epicardial coronary arteries alone were not sufficient to account for the decrease in blood flow and were most likely due to the constriction of the small resistance vessels. This mechanism is supported by studies that have shown that the function of resistance vessels is impaired in atherosclerosis. ${ }^{42}$ There is also evidence that inappropriate constriction of small diameter distal vessels, rather than the proximal large diameter coronary arteries can cause myocardial ischaemia. ${ }^{44} 45$

Increased constriction of coronary microvasculature causing a reduction in coronary blood flow in syndrome $\mathrm{X}$ was first proposed by Kaski et al..$^{46}$ In their study of 19 patients with syndrome $\mathrm{X}$ they showed that myocardial ischaemia often develops during daily life in these patients and that silent ischaemia is an important component of this syndrome. Half of the episodes of ST depression found in their study occurred at a heart rate well below that at the onset of ischaemia during exercise testing. Their findings suggested that an increased oxygen demand in the presence of an impaired coronary flow reserve was not the only cause of myocardial ischaemia in patients with syndrome $X$ and dynamic mechanisms may reduce coronary blood flow transiently as a result of increased constriction of the microvasculature.

The significant reduction in coronary blood flow velocity on hyperventilation and mental stress tests in our study was not associated with a significant change in the diameter of the left anterior descending coronary artery. This excludes epicardial coronary artery spasm and suggests that the reduction in coronary blood flow velocity may have occurred as a result of an increase in microvascular resistance and hence a reduc- tion in coronary blood flow and chest pain. This further supports the mechanism proposed by Kaski et al. ${ }^{46}$

A mechanism by which mental stress or hyperventilation could cause an increase in microvascular resistance is suggested by the changes in catecholamine concentrations found in our study. There was a significant increase in the arterial noradrenaline concentrations with both hyperventilation and mental stress. Adrenaline concentrations were also increased significantly in patients who experienced chest pain on hyperventilation. The increased catecholamine concentrations may have caused an increase in microvascular resistance leading to a reduction in coronary blood flow. Alternatively, or in addition, a local release of other vasoconstrictor substances is also a possibility. Vasoconstrictor substances such as neuropeptide $Y$ and endothelins have recently been identified that can cause myocardial ischaemia at low doses in the normal coronary circulation by reducing coronary blood flow. ${ }^{47} 48$ These substances produce ischaemia predominantly by small vessel coronary constriction. No significant change in diameter of epicardial coronary arteries was found during maximal reduction of flow in these studies. This provides evidence that distal coronary vessels have the potential to constrict to such an extent as to completely overpower metabolic autoregulation.

We did not perform coronary flow reserve studies in our patients and constrictor responses to ergonovine were also not studied. It has been shown previously, in a subset of patients with syndrome $\mathrm{X}$, that dipyridamole administration resulted in a higher minimum coronary resistance in those patients who had a positive microvascular constrictor response to ergonovine than in those patients without. ${ }^{19} 49$ These studies suggest that there may be heightened sensitivity of the coronary microcirculation to vasoconstrictor stimuli associated with an impaired microvascular vasodilator capacity in syndrome $\mathrm{X}$, in a manner similar to the racial differences found in responsiveness to recognised vasoconstrictive influences. ${ }^{50}$ Motz et al have recently shown endothelial dysfunction in coronary resistance vessels in patients with syndrome X. ${ }^{17}$ It has also been shown that endothelial dysfunction leads to increased coronary constriction to catecholamines in patients with early atherosclerosis. ${ }^{51}$ Similar mechanisms may also be contributory in some patients with syndrome $\mathrm{X}$.

An increased sympathetic drive in syndrome $\mathrm{X}$ has been suggested on the basis of an increased mean heart rate during 24 hour ambulatory electrocardiographic monitoring, increased ventricular contractility, and a rapid rise of rate-pressure product during exercise. ${ }^{5253}$ Bugiardini et al have also reported that propranolol significantly reduced ischaemic ST segment changes during ambulatory monitoring (most are preceded by an increase in heart rate) compared with placebo, an effect not seen with verapamil. ${ }^{54}$ They 
concluded that heightened sympathetic activity might account for the ambulatory ST segment responses. Camici et al have reported recently that $a_{1}$ adrenoceptors might play a part in the reduction of coronary flow reserve in patients with syndrome X..$^{55}$ Also, Romeo et al have reported that patients with syndrome $\mathrm{X}$ who show an increased sympathetic drive during exercise may develop hypertension during follow up suggesting the existence of different subgroups. ${ }^{56}$ Possible evidence against the hypothesis of an increased sympathetic drive is provided by Galassi et al. ${ }^{57}$ Their study of 12 patients with syndrome $\mathrm{X}$ failed to confirm that $a$ adrenoceptor mediated mechanisms may provoke transient myocardial ischaemia in syndrome $\mathrm{X}$.

Recently, Kaski et al have also shown that syndrome $\mathrm{X}$ does not involve increased vasomotor tone of the larger epicardial coronary arteries. ${ }^{58}$ They compared the angiographic responses of the coronary arteries to glyceryl trinitrate and ergonovine maleate in 12 patients with syndrome $X, 17$ patients with coronary artery disease, and 10 control subjects with atypical chest pain. Their results indicate that the reactivity of the large epicardial vessels to nitrates and ergonovine in syndrome $\mathrm{X}$ seems to be within the physiological range. These findings are consistent with the previous suggestion that an abnormal tone of the prearteriolar coronary vessels may be responsible for the impaired coronary flow reserve found in some patients with syndrome X. ${ }^{19} 20$ The changes found in coronary blood flow velocity in the absence of any changes in the diameter of the epicardial coronary arteries in our study lend further support to these findings, but this result is in conflict with reports of variable epicardial responses in patients with syndrome $\mathrm{X} .{ }^{22}$ This supports the hypothesis put forward by Maseri, et al, however, that an uneven distribution of small vessel vasoconstrictor zones may explain the variety of vasomotor responses in patients with syndrome $\mathrm{X} .^{20}$

The absence of significant electrocardiographic changes is puzzling, although patients experienced their typical chest pain and there was also a significant reduction in coronary blood flow velocity. It may be that the changes found in coronary blood flow were not enough to cause electrocardiographic changes although they were sufficient to cause ischaemia and pain and could be readily detected by the intracoronary Doppler probe. Maseri et al have suggested that a compensatory release of adenosine as a result of the prearteriolar reduced vasodilator capacity might cause chest pain even in the absence of myocardial ischaemia or might exacerbate the pain in the presence of myocardial ischaemia. ${ }^{20}$ It is also of note that in all cases the coronary blood flow velocity returned to normal within five minutes of stopping the tests although some of the patients continued to experience chest pain for some time after that. Thus there may not have been any electrocardiographic changes due to the short duration of the blood flow changes. This would be in keeping with the common finding that even complete occlusion of the coronary artery with a balloon during percutaneous angioplasty may not produce any electrocardiographic changes until well after one minute, although the patients complain of angina. Rozanski et al who have shown a causal association between acute mental stress and myocardial ischaemia in patients with coronary artery disease have also emphasised the importance of using a sensitive tool to detect ischaemia. ${ }^{36}$ In their study, radionuclide ventriculography was much more sensitive than electrocardiography in detecting ischaemia induced by mental stress.

The results of the oesophageal manometry study showed oesophageal dysfunction in five $(17 \%)$ of the 29 patients, which is comparable with some of the recent studies. ${ }^{6061}$ It is known that pain of an oesophageal origin may be indistinguishable from cardiac pain..$^{62}$ It is interesting that all the patients with evidence of oesophageal dysfunction did not experience chest pain with hyperventilation or mental stress. This underlines the heterogeneity of patients with syndrome $\mathrm{X}$ in whom several pathophysiological mechanisms may be operative in causing their chest pain.

One limitation of our study is the lack of a control group; the patients acted as their own controls. It is not possible to conduct such a study with the invasive technique of Doppler velocity measurements in normal subjects due to ethical considerations. Previously it has been shown in a study with six normal subjects that voluntary hyperventilation caused a decrease in mean coronary sinus oxygen content associated with a decrease in coronary flow. ${ }^{63}$ In this study coronary blood flow was assessed by a nitrous oxide method. The effect of hyperventilation on coronary blood flow was variable. Data were incomplete in one patient, and one had atrial fibrillation. It may be that coronary blood flow can be affected in normal subjects by hyperventilation and mental stress. These changes, however, may be greater in patients with syndrome $\mathrm{X}$ due to the heightened sensitivity of their coronary microcirculation to vasoconstrictor stimuli or the changes may be pronounced enough to cause ischaemia in the presence of a reduced coronary reserve.

Some patients in our study were also taking $\beta$ blockers and a 48 hour wash out period may not have been sufficient to clear the drug from the body. It is unlikely, however, that the concentrations of the drugs would have changed significantly during the course of the study.

In conclusion our study has shown that both hyperventilation and mental stress can produce chest pain in patients with syndrome $\mathrm{X}$ associated with a reduction in coronary blood flow. The results of our study suggest that the reduction in coronary flow occurs because of an increase in microvascular resistance.

We thank staff of the $x$ ray and cardiac technical departments at Papworth Hospital for their support. A C is a British Heart Foundation junior research fellow. 
1 Kemp HG, Vokonas PS, Cohn PF, et al. The anginal syndromes associated with normal coronary arteriograms: report of a six

2 Proudfit WL, Shirley EK, Jones FM. Selective cine coronary arteriography: correlation with clinical findings in 1000 cases. Circulation 1986;33:901-10.

3 Kemp HG. Left ventricular function in patients with the anginal syndrome and normal coronary arteriograms. Am 7 Cardiol 1973;32:375-6.

4 Opherk SD, Schuler G, Wetterauer K, et al. Four-year follow-up study in patients with angina pectoris and normal coronary arteriograms ("syndrome X"). Circulation 1989;80:1610-6.

5 Cannon RO, Dilsizian V, Correa R, Epstein SE, Bonow $R$. Chronic deterioration in left ventricular function in patients with microvascular angina [abstract]. $\mathcal{F} \mathrm{Am}$ Coll Cardiol 1991;17:28A.

6 Boudoulas H, Cobb TC, Leighton RF, et al. Myocardial lactate production in patients with angina-like chest pain and angiographically normal coronary arteries and left ventricle. $A m$ f Cardiol 1974;34:501-5.

7 Mammohan Singh, Parker JO. Angina pectoris with normal coronary arteriograms: Haemodynamic and metabolic response
$1975 ; 90: 555-61$.

8 James TN. Angina without coronary disease. Circulation 1970;42:189-91.

9 Elliot RS, Bratt G. The paradox of myocardial ischaemia and necrosis in young women with normal coronary arteriograms: relation to abnormal haemoglobin-oxygen dissociation. Am F Cardiol 1969;23:633-8.

10 Bass, C, Wade C, Hand D, et al. Patients with angina with normal and near normal coronary arteries: clinical and psychosocial state 12 months after angiography. $B M F$ 1983;287:1505-8.

11 Cannon RO, Quyyumi AA, Schenke WH, et al. Abnormal cardiac sensitivity in patients with chest pain and normal coronary arteries. $\mathcal{F} \mathrm{Am}$ Coll Cardiol 1990; 16:1359-66.

12 Rosano GMC, Lindsay DC, Kaski JC, Sarrel PM, PooleWilson PA. Syndrome $X$ in women: the importance of the ovarian hormones [abstract]. $¥ \mathrm{Am}$ Coll Cardiol the ovarian

13 Poole-Wilson PA. Potassium and the heart. Clinical Endocrinology and Metabolism 1984;13:249-69.

14 Cannon RO, Camici PG, Epstein SE. Pathophysiological dilemma of syndrome X. Circulation 1992;85:883-92.

15 Mosseri M, Yarom R, Gotsman MS, et al. Histologic evidence for small-vessel coronary disease in patients with angina pectoris and patent large coronary arteries. Circulation 1986;74:964-72.

16 Bugiardini R. Epicardial coronary artery reactivity in patients with syndrome $\mathrm{X}$ : the role of increased adrenergic tone. Coronary Artery Disease 1992;3:547-54.

17 Motz W, Vogt M, Rabenay O, Scheler O, Luckhoff A, Straver BE. Evidence of endothelial dysfunction in coronary resistance vessels in patients with angina pectoris and normal coronary angiograms. Am $\Im$ Cardiol toris and normal cor:

18 Dean JD, Jones CJ, Hutchison SJ, Peters JR, Henderson AH. Hyperinsulinaemia and microvascular angina ("syndrome X"). Lancet 1991;337:456-7.

19 Cannon RO, Epstein SE. "Microvascular angina" as a cause of chest pain with angiographically normal coronary arteries. $A m \mathcal{F}$ Cardiol 1988;61:1338-43.

20 Maseri A, Crea P, Kaski JC, Crake T. Mechanisms of angina pectoris in syndrome X. $\mathcal{F} \mathrm{Am}$ Coll Cardiol 1991;17:499-506.

21 Bass C, Chambers JB, Kiff P, et al. Panic anxiety and hyperventilation in patients with chest pain: a controlled study. Qf Med 1988;260:949-59.

22 Bugiardini R, Morgagni G, Pozzati A, Ottani F, Puddu P, Lenzi S. Angina with normal angiograms: a disorder involving the entire coronary tree in vasomotion (abstract). I Am Coll Cardiol 1987;9:176A.

23 Kern MJ, Deligonul U, Vandormael M, et al: Impaired coronary vasodilator reserve in the immediate postcoronary angioplasty period: analysis of coronary artery flow velocity indexes and regional cardiac venous efflux. $f$ velocity indexes and regional cardiol 1989;13:860-72.

24 Mullins PA, Chauhan A, Sharples L, et al. Impairment of coronary flow reserve in orthotopic cardiac transplant
recipients with minor coronary occlusive disease. $B r$ recipients with minor corc

25 Frayn KN, Maycock PF. Sample preparation with ionexchange resin before liquid chromatographic determination of plasma catecholamines. Clin Chem 1983;29:1426-8.

26 Wilson RF, Laughlin DE, Ackell PM, et al. Transluminal, subselective measurement of coronary artery blood flow velocity and vasodilator reserve in man. Circulation 1985;72:82-92.

27 Neill WA, Hattenhauer $M$. Impairment of myocardial $\mathrm{O}_{2}$ supply due to hyperventilation. Circulation 1975;52:854-8

28 Ardissino D, De Servi S, Barberis P, et al. Significance of hyperventilation-induced ST segment depression in patients with coronary artery disease. $7 \mathrm{Am}$ Coll Cardiol 1989;13:804-10.

29 Lewis, NP, Hutchinson SJ, Willis N, Henderson AH. Syndrome $\mathrm{X}$ and hyperventilation. $\mathrm{Br}$ Heart $\mathrm{f}$ 1991;65:94-6.

30 Nixon PGF, Freeman LJ. The "think test": a further technique to elicit hyperventilation. $\mathcal{f} R$ Soc Med
1988;81:277-9.

31 Evans DW, Lum LC. Hyperventilation: an important cause of pseudoangina. Lancet 1985;ii:826-30.

32 Chambers JB, Kiff P, Gardner WN, et al. Measurement of end-tidal pressure of carbon dioxide increases the diagnostic power of treadmill exercise testing. $B M F$ nostic power of

33 McGinn LA, White CW, Wilson RF. Interstudy variability of coronary flow reserve. Influence of heart rate, arterial pressure, and ventricular preload. Circulation 1990;81:1319-30.

34 Deanfield JE, Shea $M$, Kensett $M$, et al. Silent myocardial ischaemia due to mental stress. Lancet 1984;2:1001-5.

35 Barry J, Selwyn AP, Nabel EG, et al. Frequency of STsegment depression produced by mental stress in stable angina pectoris from coronary artery disease. $A m \mathcal{F}$ Cardiol 1988;61:989-93.

36 Rozanski A, Bairey CN, Krantz DSF, et al. Mental stress and induction of silent myocardial ischaemia in patients 1988;318:1005-12.

37 Bassan MM, Marcus HH, Ganz W. The effect of mild-tomoderate mental stress on coronary haemodynamics in patients with coronary artery disease. Circulation patients with

38 Specchia G, de Servi S, Falcone C, et al. Mental arithmetic stress testing in patients with coronary artery disease Am Heart 7 1984;108:56-63.

39 Robinson BF. Relation of heart rate and systolic blood pressure to the onset of pain in angina pectoris.

40 De Servi S, Ferrario M, Mussini A, et al. Effeti del cold pressor test e dello stress mentale sul flusso e le resistenze coronariche in pazienti con angina pectoris. G Ital Cardiol 1984;14:305-11.

41 Yeung AC, Vekshtein VI, Krantz DS. el al. The effect of atherosclerosis on the vasomotor response of coronary arteries to menta

42 Osborne J, Siegman M, Sedar A, Mooers S, Lefer A. Lack of endothelium-dependent relaxation in coronary resistance arteries of cholesterol-fed rabbits. Am $₹$ Physiol 1989;256:C591-7.

43 Sellke FW, Armstrong ML, Harrison DG. Endotheliumdependent vascular relaxation is abnormal in the coronary microcirculation of atherosclerotic primates. Circulation 1990;81:1586-93.

44 Wilson RF, Laxson DD, Lesser JR, White CW. Intense microvascular constriction after angioplasty of acute thrombotic coronary arterial lesions. Lance 1989;1:807-11

45 Pupita G, Maseri A, Kaski JC, et al. Myocardial ischaemia caused by distal coronary artery constriction in stable angina pectoris. $N$ Eng $\mathcal{F}$ Med 1990;323:514-20.

46 Kaski JC, Crea F, Nihoyannopoulos P, Hackett D, Maseri A. Transient myocardial ischaemia during daily life in patient

47 Clarke JG, Davies GJ, Kerwin $\mathrm{R}$, et al. Coronary artery infusion of neuropeptide $\mathrm{Y}$ in patients with angina pectoris. Lancet 1987;1:1057-9.

48 Larkin SW, Clarke JG, Keogh BE, et al. Intracoronary endothelin induces myocardial ischaemia by small vessel endothelin induces myocardial ischaemia by small ves

49 Cannon RO, Schenke WH, Leon MB, Rosing DR Urquhart J, Epstein SE. Limited coronary flow reserve Urquhart J, Epstein SE. Limited coronary flow reserve after dipyridamole in patients with ergonovine-induced

50 Maseri A, Kaski JC. Pathogenetic mechanisms of coronary artery spasm. $\mathcal{f}$ Am Coll Cardiol 1989;14: 610-12.

51 Vita JA, Treasure CB, Fish D, et al. Endothelial dysfunction leads to increased coronary constriction to catecholamines in patients with early atherosclerosis. [abstract]. $\mathcal{A} \mathrm{Am}$ Coll Cardiol 1990;15:158A

52 Romeo F, Gaspardone A, Ciavolella M, Gioffer P, Reale A. Verapamil versus acebutol for syndrome $\mathrm{X}$. $A m \mathcal{F}$ Cardiol 1988;62:312-3.

53 Galassi AR, Kaski JC, Crea F, et al. Heart rate response during exercise testing and ambulatory ECG monitoring in patients with syndrome X. Am Heart f 1991; in patients

54 Bugiardini R, Borghi A, Biagetti L, Puddu P. Comparison of verapamil versus propranolol therapy in syndrome $\mathrm{X}$. of verapamil versus propranolol

55 Camici PG, Marraccini P, Gistri R, Lorenzoni R, Sorace $O$, L'Abbate A. Alpha-1 adrenergic tone and coronary reserve in patients with syndrome $X$ [abstract]. Circulation 1991;84:1686.

56 Romeo F, Rosano GC, Rosan V, Martuscelli E, Micelli F, Lomardo $\mathrm{L}$, et al. The clinical evolution of patient diagnosed with syndrome $\mathrm{X}$ during a five year follow up [abstract]. $\mathcal{F} \mathrm{Am}$ Coll Cardiol 1992;19:87A

57 Galassi AR, Kaski JC, Pupita G, Vejar M, Crea F, Maser A. Lack of evidence for alpha-adrenergic receptormediated mechanisms in the genesis of ischaemia in syndrome X. Am 7 Cardiol 1989;64:264-9.

58 Kaski JC, Tousoulis D, Galassi AR, et al. Epicardial coronary artery tone and reactivity in patients with normal coronary arteriograms and reduced coronary flow reserve (syndrome X). ₹ Am Coll Cardiol 1991;18:50-4.

59 Montorsi $\mathrm{P}$, Manfredi $\mathrm{M}$, Loaldi A, et al. Comparison of coronary vasomotor responses to nifedipine in syndrome 1989;63:1198-202. 
60 Cannon RO, Cattau EL, Yakshe PN, et al. Coronary flow reserve, esophageal motility, and chest pain in patients with angiographically normal coronary arteries. $\mathrm{Am} \mathcal{F}$ Cardiol 1990;88:217-22.

61 Peters L, Maas L, Petty D, et al. Spontaneous non-cardiac chest pain: evaluation by 24 hour ambulatory chest pain: evaluation by 24 hour ambulatory esophageal motility
62 Kramer P, Hollander W. Comparison of experimental esophageal pain with clinical pain of angina pectoris and esophageal disease. Gastroenterology 1955;29. 719-43.

63 Rowe GG, Castillo CA, Crumpton CW. Effects of hyperventilation on systemic and coronary haemodynamics. Am Heart $¥$ 1962;63:67-77.
Heart disease is now the third commonest cause of maternal mortality in the United Kingdom, after hypertension and thromboembolism. The data of the Report on Confidential Enquiries into Maternal Deaths in the United Kingdom 1985 to $1987^{1}$ show that the number of deaths has been constant (23 deaths between 1985 and 1987, about 1 per 100000 births) since 1973. During this time deaths from other causes such as anaesthesia and haemorrhage have become less common, making cardiac disease relatively more important. Between 1973 and 1987, the number of cardiac deaths from congenital heart disease has doubled whereas the number of deaths from acquired heart disease has halved.

Of the 10 patients who died of congenital heart disease, three had endocarditis (not contracted in labour) which was a major feature of their terminal illness and the remainder had pulmonary hypertension, either Eisenmengers' syndrome (four cases) or "primary" pulmonary hypertension (three cases). In the patients with acquired heart disease nine died from coronary artery disease, two had endocarditis, one had "myocarditis", and the other had cardiomyopathy (probably puerperal).

What lessons can be learned from these figures? The patients who died from coronary artery disease were often older, smokers, and might have died whether or not they were pregnant. As contributing factors to cardiac mortality the authors of the series of confidential enquiries cited the failure of patients to comply with doctors' advice, the failure of patients to be seen regularly in joint consultation between physicians and obstetricians and the general failure of cooperation between obstetricians, cardiologists, and anaesthetists. We should not become complacent and lose sight of these problems.

But pulmonary hypertension remains the major maternal risk. Even if the patients are treated with full coopera- tion between experts, we do not know how best to man- $\vec{\oplus}$ age pulmonary hypertension in pregnancy apart from $\omega$ advising patients not to become pregnant or urging termination. On the other hand, unless patients with pulmonary hypertension are seen in specialist clinics there will be no advances in management. The ability to induce selective pulmonary artery vasodilatation would be particularly valuable in pregnancy.

Physicians need to be particularly vigilant about endocarditis in pregnancy. Perhaps the immunosuppression that allows survival of the fetal homograft and increases the risk of viral illness also increases susceptibility to $\overrightarrow{\widetilde{\Phi}}$ endocarditis.

Finally, there is insufficient awareness of the condition of puerperal cardiomyopathy among non-cardiologists (obstetricians and general practitioners) treating women $\delta$ in pregnancy. Any patient presenting with breathlessness at the end of pregnancy must be adequately evaluated. 옥 The chest $x$-ray is the screening investigation that is usu- $D$ ally omitted because of groundless fears of the radiation hazard to the fetus. Most, though not all, patients with $N_{\circ}$ puerperal cardiomyopathy have a cardiac silhouette that $N$ is enlarged even by pregnancy standards and that would $\mathrm{N}$ prompt further investigation by echocardiography. When ${ }_{\sigma}$ puerperal cardiomyopathy presents antenatally the patient should be delivered and treated with anticoagulants because of the risk of thromboembolism. Without $\stackrel{\Phi}{+}$ heart transplantation, however, a third of these patients will die.

Queen Charlotte's and Chelsea Hospitals, Goldhawk Road,

London W6 OXG

\section{MICHAEL DE SWIET}

Department of Health and Social Security Report on Confidential 8 Enquires into Maternal Deaths in England and Wales, 1985-1987. HMSO, 1991. 